\title{
Leisure-Time Physical Activity and All-Cause Mortality
}

\author{
Jouni Lahti*, Ansku Holstila, Eero Lahelma, Ossi Rahkonen
}

Hjelt Institute, Department of Public Health, University of Helsinki, Helsinki, Finland

\begin{abstract}
Background: Physical inactivity is a major public health problem associated with increased mortality risk. It is, however, poorly understood whether vigorous physical activity is more beneficial for reducing mortality risk than activities of lower intensity. The aim of this study was to examine associations of the intensity and volume of leisure-time physical activity with all-cause mortality among middle-aged women and men while considering sociodemographic and health related factors as covariates.

Methods: Questionnaire survey data collected in 2000-02 among 40-60-year-old employees of the City of Helsinki $(\mathrm{N}=8960)$ were linked with register data on mortality (74\% gave permission to the linkage) providing a mean follow-up time of 12 -years. The analysis included 6429 respondents (79\% women). The participants were classified into three groups according to intensity of physical activity: low moderate, high moderate and vigorous. The volume of physical activity was classified into three groups according to tertiles. Cox regression analysis was used to calculate hazard ratios (HR) and $95 \%$ confidence intervals (Cls) for all-cause mortality.

Results: During the follow up 205 participants died. Leisure-time physical activity was associated with reduced risk of mortality. After adjusting for covariates the vigorous group $(\mathrm{HR}=0.54,95 \% \mathrm{Cl} 0.34-0.86)$ showed a reduced risk of mortality compared with the low moderate group whereas for the high moderate group the reductions in mortality risk $(H R=0.72$, $95 \% \mathrm{Cl}$ 0.48-1.08) were less clear. Adjusting for the volume of physical activity did not affect the point estimates. Higher volume of leisure-time physical activity was also associated with reduced mortality risk; however, adjusting for the covariates and the intensity of physical activity explained the differences.
\end{abstract}

Conclusions: For healthy middle-aged women and men who engage in some physical activity vigorous exercise may provide further health benefits preventing premature deaths.

Citation: Lahti J, Holstila A, Lahelma E, Rahkonen O (2014) Leisure-Time Physical Activity and All-Cause Mortality. PLoS ONE 9(7): e101548. doi:10.1371/journal. pone.0101548

Editor: Yang-Ching Chen, TaipeiCityHospital, Taiwan

Received February 21, 2014; Accepted June 9, 2014; Published July 2, 2014

Copyright: (c) 2014 Lahti et al. This is an open-access article distributed under the terms of the Creative Commons Attribution License, which permits unrestricted use, distribution, and reproduction in any medium, provided the original author and source are credited.

Funding: The study was funded by the University of Helsinki (www.helsinki.fi/university/), theMinistry of Education and Culture (www.minedu.fi/OPM/?lang = en) grant \#6303383, the Juho Vainio Foundation (www.juhovainionsaatio.fi/pages/in-english/home.php) grant \#4702850 and the Academy of Finland (www.aka.fi/ en-GB/A/) grant \#1257362. The funders had no role in study design, data collection and analysis, decision to publish, or preparation of the manuscript.

Competing Interests: The authors have declared that no competing interests exist.

*Email: jouni.mm.lahti@helsinki.fi

\section{Introduction}

There is an extensive amount of studies showing that physical activity is important to health benefits and reducing mortality risk [1]. Some studies suggest that avoiding physical inactivity is the most important [2], [3] and that a higher volume of physical activity further reduces mortality risk indicating a dose-response association [3]. In addition, a recent review and meta-analysis suggested a dose-response association between non-vigorous physical activity and mortality [4]. Another recent review and meta-analysis showed that vigorous intensity physical activity is associated with even larger reductions in mortality risk than activities of lower intensity [5]. However, these findings may be related to the higher volume of activity and not the intensity per se. Thus, it is still poorly understood whether vigorous intensity physical activity is more beneficial for reducing mortality risk than activities of lower intensity [5].

As those who engage in vigorous physical activity may have a higher volume of physical activity and thus lower the risk of mortality, the volume of physical activity needs to be considered in the analyses to separate the independent contribution of vigorous activity on reduced mortality risk. In addition, in epidemiological studies examining physical activity and mortality, existing physical as well as mental health differences need to be considered in the analysis because health problems potentially affect physical activity [6]. Furthermore, it is also important to take into account other covariates that are risk factors for mortality and may be unequally distributed between physical activity groups [7], for example socioeconomic position [8], [9], BMI, smoking and drinking problems [8], [10].

The aim of this study was to examine the associations of the intensity and volume of leisure-time physical activity with all-cause mortality among middle-aged women and men while considering sociodemographic and health related factors as covariates. Additional aim was to examine the independent association of the intensity of leisure-time physical activity by adjusting for the volume of leisure-time physical activity in the analyses and vice versa for the volume of leisure-time physical activity. 


\section{Materials and Methods}

\section{Ethics statement}

The study is approved by the ethics committees of the Department of Public Health, University of Helsinki and the health authorities of the City of Helsinki. Informed, written consent was obtained from all participants in the study.

\section{Data sources}

Helsinki Health Study baseline questionnaire surveys were conducted in 2000, 2001 and 2002 among 40- to 60-year-old employees of the City of Helsinki. The sample consisted of 13346 persons and 7148 women and 1799 men returned the questionnaire (response rate 67\%). Younger employees, men and manual workers were somewhat underrepresented among the respondents, but otherwise the data satisfactorily represent the target population [11], [12].

Mortality data were obtained from the registers of Statistics Finland. All deaths until the end of year 2012 were individually linked with the questionnaire data $(74 \%$ gave permission to the linkages), providing a mean follow-up time of 12-years. There was missing information in some study variables $(n=149)$. Those, reporting no leisure-time physical activity $(n=28)$ were excluded from the analyses. This yielded 5051 women and 1378 men for the analyses.

\section{Leisure-time physical activity}

The respondents were asked their average weekly hours of leisure-time physical activity/exercise (including commuting) within the previous 12 months. The respondents were instructed first to estimate the intensity of each activity they engaged in and then to estimate how many hours per week they spent on average in physical activity corresponding to each grade of intensity. Four grades of intensity were given exemplified by common activities: walking, brisk walking, jogging, and running, or their equivalent activities. Each intensity grade had five response alternatives: no activity, 0-1/2 hours/week, 1/2-1 hours/week, 2-3 hours/week, $\geq$ 4 hours/week. We classified the participants into three groups according to the highest intensity of physical activity they engaged in: low moderate (e.g. walking), high moderate (e.g. brisk walking), and vigorous (e.g. jogging/running). The volume of physical activity was assessed by approximate metabolic equivalents (MET) [13]. MET-hours per week were calculated by multiplying the time used (weekly hours) by the estimated MET value of each physical activity grade [14]' and then adding the four values together. The volume of leisure-time physical activity was classified into three groups according to tertiles (cutpoints: 14.75 and 34.75 MET-hours/wk) [15].

\section{Covariates}

Age included five groups at baseline: 40, 45, 50, 55 and 60 years. Smoking was categorized into non-smokers, ex-smokers and smokers. Drinking problems were measured by the CAGE questionnaire [16]. Four occupational social classes were used as an indicator of socioeconomic position (SEP): managers (managerial and administrative work) and professionals (e.g. teachers and doctors), semi-professionals (e.g. nurses and foremen), routine nonmanual employees (e.g. child minders and assistant maids), and manual workers (e.g. transport and cleaning work) [17]. The respondents were asked how strenuous their work is physically and mentally. Physical strenuousness of work was dichotomized as heavy or light. The same procedure was repeated for mental strenuousness of work. Body mass index (BMI) was calculated, using questionnaire data on height $(\mathrm{m})$ and weight $(\mathrm{kg})$. Physical functioning was measured by the physical functioning sub-scale of the Short-Form 36 (SF-36) health questionnaire [18]. Mental health was measured by the general mental health sub-scale of the SF-36. These SF-36 scores range from zero to 100 , higher scores indicate better health, and mean scores between $45-54$ years are 84.6 and 75.3 for physical functioning and mental health, respectively [18]. Details of limiting longstanding illness (LLI) were requested in two separate questions and classified into two groups: those with and those without LLI.

\section{Statistical methods}

First descriptive statistics were calculated. Cox regression analysis was used to calculate hazard ratios (HR). In model 1 age and gender were adjusted for. In model 2 additional adjustments were made for smoking, drinking problems, occupational social class, physical and mental strenuousness of work. In model 3 additional adjustments were made for BMI, physical functioning, mental health and LLI. In model 4 the HRs were further adjusted for the volume or the intensity of leisure-time physical activity. The proportional hazards assumption was examined using Schoenfeld residuals [19] and confirmed for leisure-time physical activity and covariates. SPSS 20.0 statistical package was used.

\section{Results}

Table 1 presents the distributions of baseline study variables (Table 1). Increasing intensity and volume both showed younger age. Women were less often vigorously active than men but in the volume groups gender differences were smaller. Increasing intensity and volume also showed less smoking but differences in drinking problems were minor and somewhat inconsistent. Increasing intensity was associated with higher SEP but between the volume groups there were smaller SEP differences. There were some differences in the physical and mental strenuousness of work. Increasing intensity as well as volume was associated with lower BMI, better physical functioning, mental health and lower prevalence of LLI. Physical activity intensity and volume were inter-related. Over half of the vigorously active was in the highest third of physical activity volume whereas less than every tenth was in the lowest third. Of the high moderate group, the pattern was different; less than a quarter was in the highest third and over forty percent in the lowest third. Of the low moderate group, almost eighty percent were in the lowest third whereas none were in the highest third.

During the follow up 205 (2.6\% of women and $5.2 \%$ of men) participants died (Table 2). According to the intensity of leisuretime physical activity, those engaging in low moderate-intensity activities $(6.0 \%)$ had the highest mortality rate and those engaging in vigorous activities $(2.2 \%)$ had the lowest rate. For the high moderate-intensity group the mortality rate was $3.4 \%$. According to the volume of leisure-time physical activity, the lowest third had the highest mortality rate $(4.3 \%)$, whereas for the highest $(2.7 \%)$ and middle thirds $(2.5 \%)$ the mortality rates were somewhat lower. More than half of all deaths were cancer related (ICD10 codes C00-D48). Among women breast cancer was the most common cancer causing death whereas among men the most common cancer was lung cancer. Cardiovascular disease (ICD-10 codes I00-I99) was the second common diagnostic group causing one fourth of the deaths. Among both women and men the most common cardiovascular disease was arteriosclerotic vascular disease.

Firstly, we examined the association of the three intensity grades of leisure-time physical activity with mortality adjusting for 


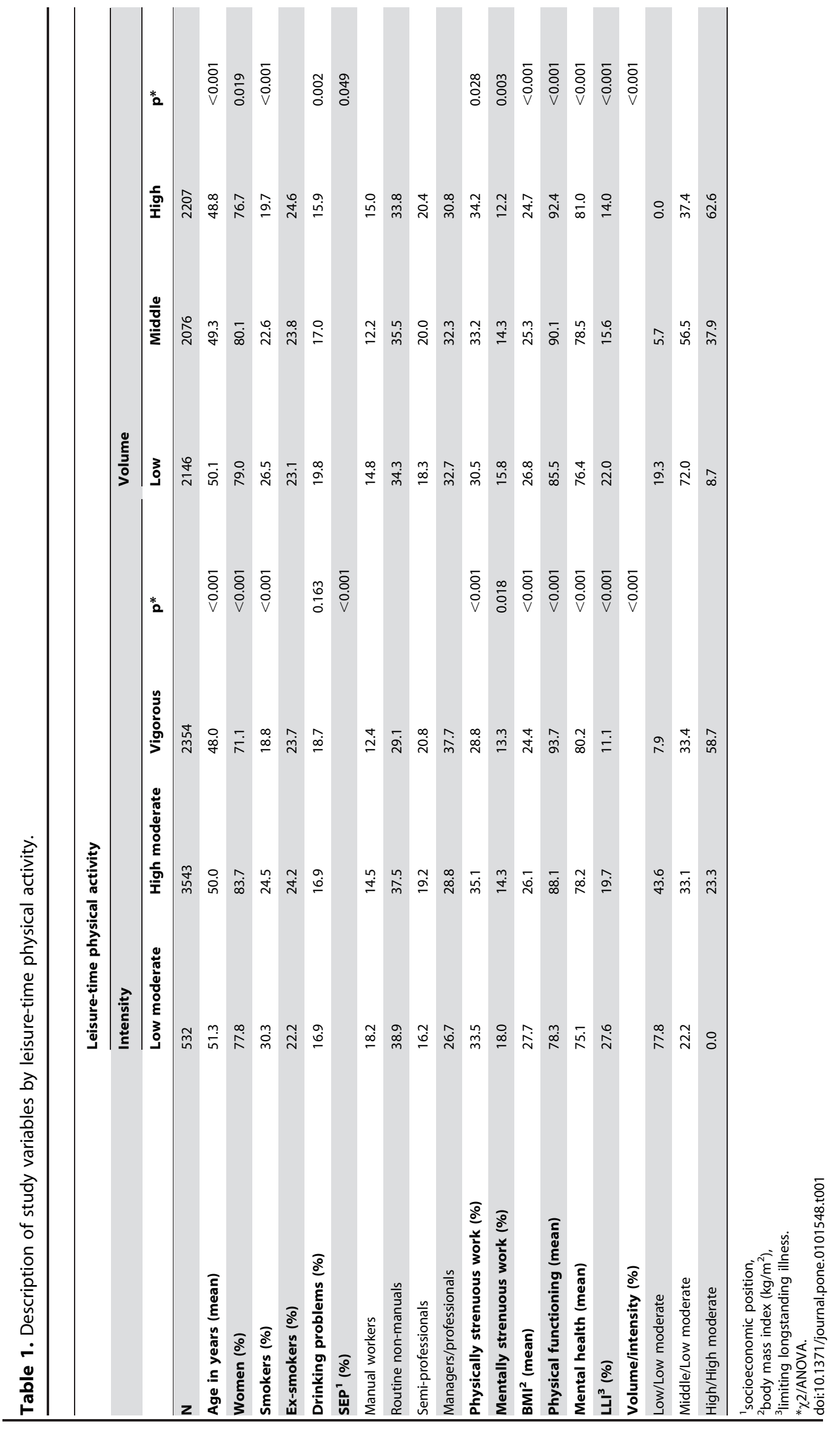




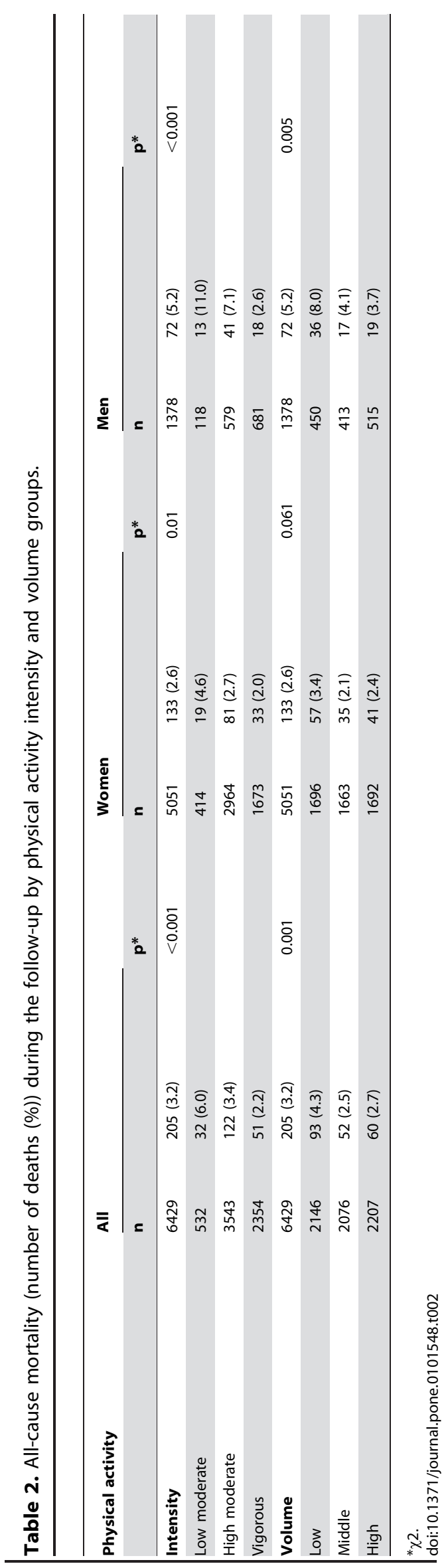

covariates and the volume of leisure-time physical activity and using Cox regression analyses. The associations between the intensity of leisure-time physical activity and mortality were similar for women and men $(\mathrm{p}=0.2$ for interaction) and women and men were pooled for the analyses. Compared with the low moderate group, the vigorous ( $\mathrm{HR}=0.47,95 \%$ CI $0.30-0.73$ ) group had clearly reduced mortality risk when age, gender and other confounders were adjusted for in model 2 (Table 3). Also the high moderate group had reduced mortality risk $(\mathrm{HR}=0.66,95 \%$ CI 0.45-0.98). When additional adjustments were made for BMI, physical functioning, mental health and LLI the associations attenuated and remained for the vigorous group $(\mathrm{HR}=0.54,95 \%$ Cl 0.34-0.86) but lost statistical significance for the high moderate group. Adjusting for the volume of leisure-time physical activity had no effects on the point estimates.

We then examined the contribution of the volume of leisuretime physical activity classified into three groups according to tertiles. The associations were similar for women and men $(p=0.5$ for interaction) and women and men were pooled for the analyses. Compared with the lowest third, the middle $(\mathrm{HR}=0.61,95 \%$ CI $0.44-0.86)$ and the highest $(\mathrm{HR}=0.67,95 \%$ CI $0.48-0.93)$ thirds had reduced mortality risk when age and gender were adjusted for (Table 4). When additional adjustments were made for other confounders in model 2, the association lost statistical significance for the highest third. Further adjustments for BMI, physical functioning, mental health and LLI attenuated the associations and statistical significance was lost for both the middle $(\mathrm{HR}=0.71$, 95\% CI $0.50-1.00)$ and the highest (HR $=0.81,95 \%$ CI $0.58-$ 1.14) thirds. Adjusting for the intensity of leisure-time physical activity further attenuated the associations and no clear patterns remained.

\section{Discussion}

We aimed to examine whether the intensity and volume of leisure-time physical activity were associated with all-cause mortality among middle-aged employees after considering key covariates. Increasing intensity and volume of leisure-time physical activity was associated with reduced mortality risk similarly among women and men. Those engaging in vigorous activity had clearly reduced mortality risk compared with those engaging in low moderate-intensity activity, whereas, high moderate-intensity activity such as brisk walking showed weaker associations. The volume of leisure-time physical activity did not explain the reduced risk found for the vigorous intensity physical activity. The higher volume of leisure-time physical activity was also associated with reduced mortality risk; however, adjusting for the covariates and the intensity of physical activity explained the differences.

The findings of this study suggest that vigorous activity in particular is associated with reduced mortality independently of the volume of leisure-time physical activity and other covariates. Few previous studies have examined the intensity of physical activity controlling for the volume of physical activity using different methods and health outcomes [15], [20]-[23]. In general, the results of these studies are in accordance with our study. Only one previous study has examined the intensity of physical activity and mortality [20]. The study by Lee and Paffenbarger was conducted only among men and showed that vigorous physical activity was clearly associated with reduced mortality risk and moderate-intensity activity showed some, albeit less clear reductions in mortality risk [20]. Another study among men showed that vigorous physical activity reduced the risk of coronary heart disease but moderate-intensity activity showed weaker associations [21]. A cross sectional study using objective physical activity 
Table 3. Hazard ratios $(95 \% \mathrm{Cl})$ for all-cause mortality according to the intensity of leisure-time physical activity.

\begin{tabular}{llll}
\hline \multicolumn{4}{l}{ HR (95\%Cl) according to the intensity of physical activity } \\
\hline & Low moderate & High moderate & Vigorous \\
\hline Model 1 & 1.00 & $0.64(0.44-0.95)$ & $0.43(0.27-0.67)$ \\
Model 2 & 1.00 & $0.66(0.45-0.98)$ & $0.47(0.30-0.73)$ \\
Model 3 & 1.00 & $0.72(0.48-1.08)$ & $0.54(0.34-0.86)$ \\
Model 4 & 1.00 & $0.73(0.49-1.11)$ & $0.55(0.32-0.94)$ \\
\hline
\end{tabular}

Model 1 adjusted for age and gender.

Model 2 adjusted for covariates in model 1 and additionally for occupational social class, physical and mental strenuousness of work, smoking and drinking problems. Model 3 adjusted for covariates in model 2 and additionally for body mass index, physical functioning mental health and limiting longstanding illness.

Model 4 adjusted for covariates in model 3 and additionally for the volume of physical activity.

doi:10.1371/journal.pone.0101548.t003

measurement showed that vigorous physical activity was associated with reduced risk of metabolic syndrome independently of the physical activity volume among women and men [23]. Also our previous studies have showed that vigorous activity is more beneficial than moderate-intensity physical activity for various domains of health including sickness absence [15] and physical health functioning [22].

Previous studies have suggested a dose-response association between physical activity volume and mortality [3]. Some studies, however, suggest that higher amounts of activity do not further reduce mortality risk [2] which is in accordance with our results. However, in our study the association of the volume of leisure-time physical activity with mortality was not as strong as for the intensity, and the association was explained by the intensity of physical activity and other covariates. It should be noted that in addition to the intensity and volume also the type of physical activity may be important for health benefits and reduced mortality [2], [24].

There are some methodological issues when examining the independent associations of the intensity and volume of physical activity with health outcomes. The intensity and volume of physical activity are inter-related as the volume (MET-hours/wk) is the product of intensity and time spent in physical activity. Furthermore, those who engage in vigorous physical activity are also likely to engage in lower intensity activity. In these data, less than five per cent of the vigorously active did not engage in high or low moderate activities (data not shown). There are different methods, described in previous studies [15], [20]-[23], to examine the associations of different intensity physical activity with various health outcomes, independent of the volume of physical activity.
We used multivariate models similar to study by Tanasescu et al. [21]. A statistical problem may, however, arise in multivariate models if highly correlated (correlation coefficient $>0.60$ ) variables are in the same model i.e. multicollinearity. When we examined the intensity of physical activity, adjusting for the volume of physical activity slightly widened the confidence intervals which may indicate multicollinearity. However, the correlation between the intensity and volume of physical activity was moderate (correlation coefficient $=0.52$ ) and thus should not pose a statistical problem in the analyses.

When examining physical activity and mortality taking account of baseline health is important as existing health problems may restrict physical activity in general and, vigorous activity in particular. This has been somewhat ignored in previous studies [20]. We adjusted extensively for baseline health related covariates including BMI, LLI, physical functioning and mental health. For example, the SF-36 physical functioning subscale indicates health problems that affect physical functioning such as participation in physical activities. These factors may be partial mediating factors between leisure-time physical activity and mortality and there is a risk of overadjustment. Our sensitivity analyses showed that of the covariates included, physical functioning attenuated the examined associations the most but the attenuating effect was relative small and the associations remained strong for the vigorous group even after all adjustments.

Regarding the highest intensity of leisure-time physical activity less than every tenth reported only low moderate-intensity physical activity such as walking. About half reported high moderateintensity activity such as brisk walking and more than every third vigorous activity such as jogging or running. Men engaged in

Table 4. Hazard ratios $(95 \% \mathrm{Cl})$ for all-cause mortality according to the volume of leisure-time physical activity.

\begin{tabular}{|c|c|c|c|}
\hline \multicolumn{4}{|c|}{ HR $(95 \% \mathrm{Cl})$ according to the volume of physical activity } \\
\hline & Low & Middle & High \\
\hline Model 1 & 1.00 & $0.61(0.44-0.86)$ & $0.67(0.48-0.93)$ \\
\hline Model 2 & 1.00 & $0.65(0.47-0.92)$ & $0.73(0.53-1.01)$ \\
\hline Model 3 & 1.00 & $0.71(0.50-1.00)$ & $0.81(0.58-1.14)$ \\
\hline Model 4 & 1.00 & $0.78(0.55-1.12)$ & $0.99(0.67-1.47)$ \\
\hline
\end{tabular}

Model 1 adjusted for age and gender.

Model 2 adjusted for covariates in model 1 and additionally for occupational social class, physical and mental strenuousness of work, smoking and drinking problems. Model 3 adjusted for covariates in model 2 and additionally for body mass index, physical functioning mental health and limiting longstanding illness.

Model 4 adjusted for covariates in model 3 and additionally for the intensity of physical activity.

doi:10.1371/journal.pone.0101548.t004 
vigorous activity more often than women and consequently a large part of women engaged in high moderate-intensity activity. In addition, the cutpoint i.e. 14.75 MET-hours per week for the lowest third of physical activity volume is comparable to 2.5 hours of brisk walking per week (15 MET-hours) which is the base of physical activity recommendations [1]. Thus the studied cohort is relatively active physically compared with European adults in general [25]. Also vigorous activity was quite common and thus achievable for many middle-aged.

Men had a higher mortality rate compared with women which is in accordance with the general middle-aged population in Finland [26]. Among middle-aged Finns the most common cause of death is still coronary heart disease, although cardiovascular disease mortality in general has decreased. Also cancers (e.g. lung cancer among men and breast cancer among women) are common causes of death. Other common causes include alcohol related deaths and suicides [26]. In this study, the relatively low number of deaths during the follow-up did not allow detailed cause-specific analyses of mortality. However, the most common diagnostic group was cancer accounting for half of the deaths and the second common diagnostic group was cardiovascular disease accounting for one fourth of the deaths. Our sensitivity analyses showed similar patterns between leisure-time physical activity and cancer mortality as with all-cause mortality (data not shown).

A number of biological mechanisms for the association between physical activity and mortality have been proposed in previous studies [5]. Biological mechanisms proposed include e.g. increase in the high-density lipoproteins and reduction of coronary artery calcium, however, these mechanisms relate cardiovascular diseases and since in these data over half of the deaths were cancer related other mechanism needs to be considered. It has been proposed that exercise may improve anti-tumor immune defenses and antioxidant defense systems [27]. Also other mechanisms have been proposed such as reduced abdominal fat, however, in these data adjusting for BMI had only minimal effects on the association between physical activity and mortality. On the other hand, BMI does not directly measure abdominal fat. In addition, there is evidence that vigorous activity reduces the risk of colorectal cancer more than activities of lower intensity [28], however, the intensity of physical activity is not related to the risk of breast cancer although there is a dose-response association between physical activity volume and breast cancer [29]. As higher intensity of physical activity contributes to fitness more than activities of lower intensity the potential mechanisms regarding the intensity are probably at least partly related to fitness. Previous studies have shown that increased fitness at middle-age reduce the risk of mortality, comparable to smoking cessation [30]. Nonetheless, as the scientific evidence for the potential mechanisms is limited, further studies are needed.

\section{References}

1. Physical Activity Guidelines Advisory Committee (2008) Physical Activity Guidelines Advisory Committee Report. Washington, DC: U.S. Department of Health and Human Services.

2. Johnsen NF, Ekblond A, Thomsen BL, Overvad K, Tjonneland A (2013) Leisure time physical activity and mortality. Epidemiology 24: 717-725. doi:10.1097/EDE.0b013e31829e3dda.

3. Moore SC, Patel AV, Matthews CE, Berrington de Gonzalez A, Park Y, et al. (2012) Leisure time physical activity of moderate to vigorous intensity and mortality: A large pooled cohort analysis. PLoS Med 9: e1001335. doi:10.1371/ journal.pmed.1001335.

4. Woodcock J, Franco OH, Orsini N, Roberts I (2011) Non-vigorous physical activity and all-cause mortality: Systematic review and meta-analysis of cohort studies. Int J Epidemiol 40: 121-138. doi:10.1093/ije/dyq104.

5. Samitz G, Egger M, Zwahlen M (2011) Domains of physical activity and allcause mortality: Systematic review and dose-response meta-analysis of cohort studies. Int J Epidemiol 40: 1382-1400. doi:10.1093/ije/dyr112.

\section{Strengths and limitations}

We were able to take into account key health related covariates, including LLI, physical functioning and mental health which is highly important as those with health problems may be unable to engage in physical activity, especially, with higher intensity. There were only few people that did not engage in any leisure-time physical activity thus comparison with them was not possible. This study focuses on leisure-time physical activity and other activities such as household activities that contribute to energy expenditure were not considered in the analyses, which is a limitation. In addition, other aspects of leisure-time physical activity such as the type of activity were not examined. Leisure-time physical activity was self-reported and lacks formal validation. However, other questionnaire data as well as objectively measured physical activity [23] have produced similar findings and no single questionnaire has proven superior for measuring leisure-time physical activity in large surveys [31]. The use of objectively measured physical activity would have added to the study, however, in large datasets questionnaires are still the first choice of measurement. There are characteristics in our data that limit the generalizability of the findings. The participants were middle-aged municipal employees and $80 \%$ of them were women which corresponds to the gender distribution of the municipal sector in general and the staff of the City of Helsinki. We pooled women and men in the analyses and thus these results are female dominated. However, the association between physical activity and mortality was not statistically significantly different between women and men.

\section{Conclusions}

Increasing intensity and volume of leisure-time physical activity was associated with reduced mortality risk. Vigorous activity was clearly associated with reduced mortality risk independently of the volume of leisure-time physical activity. For healthy middle-aged women and men who engage in some physical activity vigorous exercise may provide further health benefits preventing premature death. Future studies examining physical activity and mortality risk should consider the intensity of physical activity more carefully.

\section{Acknowledgments}

We thank the City of Helsinki and all the members of the Helsinki Health Study group for their contribution.

\section{Author Contributions}

Conceived and designed the experiments: JL AH EL OR. Analyzed the data: AH. Wrote the paper: JL AH EL OR.

6. Roshanaei-Moghaddam B, Katon WJ, Russo J (2009) The longitudinal effects of depression on physical activity. Gen Hosp Psychiatry 31: 306-315. doi:10.1016/ j.genhosppsych.2009.04.002.

7. Laaksonen M, Prättälä R, Karisto A (2001) Patterns of unhealthy behaviour in Finland. Eur J Public Health 11: 294-300.

8. Pekkanen J, Tuomilehto J, Uutela A, Vartiainen E, Nissinen A (1995) Social class, health behaviour, and mortality among men and women in eastern Finland. BMJ 311: 589-593.

9. Stringhini S, Sabia S, Shipley M, Brunner E, Nabi H, et al. (2010) Association of socioeconomic position with health behaviors and mortality. JAMA 303: 1159 1166. doi: $10.1001 /$ jama.2010.297.

10. Khaw KT, Wareham N, Bingham S, Welch A, Luben R, et al. (2008) Combined impact of health behaviours and mortality in men and women: The EPICNorfolk prospective population study. PLoS Med 5: e12. doi:10.1371/ journal.pmed.0050012 
11. Laaksonen M, Aittomäki A, Lallukka T, Rahkonen O, Saastamoinen P, et al. (2008) Register-based study among employees showed small nonparticipation bias in health surveys and check-ups. J Clin Epidemiol 61: 900-906.

12. Lahelma E, Aittomaki A, Laaksonen M, Lallukka T, Martikainen P, et al. (2013) Cohort profile: The Helsinki health study. Int J Epidemiol 42: 722-730. doi:10.1093/ije/dys039.

13. Ainsworth BE, Haskell WL, Whitt MC, Irwin ML, Swartz AM, et al. (2000) Compendium of physical activities: An update of activity codes and MET intensities. Med Sci Sports Exerc 32: S498-504.

14. Kujala UM, Kaprio J, Sarna S, Koskenvuo M (1998) Relationship of leisuretime physical activity and mortality: The Finnish twin cohort. JAMA 279: 440444.

15. Lahti J, Laaksonen M, Lahelma E, Rahkonen O (2010) The impact of physical activity on sickness absence. Scand J Med Sci Sports 20: 191-199. doi:10.1111/ j.1600-0838.2009.00886.x.

16. Schofield A (1988) The CAGE questionnaire and psychological health. Br J Addict 83: 761-764

17. Lahelma E, Martikainen P, Rahkonen O, Roos E, Saastamoinen P (2005) Occupational class inequalities across key domains of health: Results from the Helsinki health study. Eur J Public Health 15: 504-510. doi:10.1093/eurpub/ cki022.

18. Ware JE, Snow KK, Kosinski M, Gnadek B (1993) SF-36 Health survey: manual and interpretation guide. Boston, MA: The Health Institute, New England Medical Center.

19. Schoenfeld (1982) Partial residuals for the proportional hazards regression model. Biometrica 69: 239-241

20. Lee IM, Paffenbarger RS Jr (2000) Associations of light, moderate, and vigorous intensity physical activity with longevity. The Harvard alumni health study. Am J Epidemiol 151: 293-299.

21. Tanasescu M, Leitzmann MF, Rimm EB, Willett WC, Stampfer MJ, et al. (2002) Exercise type and intensity in relation to coronary heart disease in men. JAMA 288: 1994-2000.
22. Lahti J, Laaksonen M, Lahelma E, Rahkonen O (2010) The impact of physical activity on physical health functioning-a prospective study among middle-aged employees. Prev Med 50: 246-250. doi:10.1016/j.ypmed.2010.02.007.

23. Janssen I, Ross R (2012) Vigorous intensity physical activity is related to the metabolic syndrome independent of the physical activity dose. Int J Epidemiol 41: 1132-1140. doi:10.1093/ije/dys038.

24. Sabia S, Dugravot A, Kivimaki M, Brunner E, Shipley MJ, et al. (2012) Effect of intensity and type of physical activity on mortality: Results from the whitehall II cohort study. Am J Public Health 102: 698-704. doi:10.2105/ AJPH.2011.300257.

25. Martinez-Gonzalez MA, Varo JJ, Santos JL, De Irala J, Gibney M, et al. (2001) Prevalence of physical activity during leisure time in the European Union. Med Sci Sports Exerc 33: 1142-1146.

26. Official Statistics of Finland (OSF): Causes of death [e-publication]. ISSN $=1799-5078$. Helsinki: Statistics Finland [referred: 17.9.2013]. Available: http://www.tilastokeskus.fi/til/ksyyt/kat_en.html.

27. Friedenreich GM, Orenstein MR (2002) Physical activity and cancer prevention: Etiologic evidence and biological mechanisms. J Nutr 132: 3456S-3464S.

28. Slattery ML (2004) Physical activity and colorectal cancer. Sports Med 34: 239252.

29. Monninkhof EM, Elias SG, Vlems FA, van der Tweel I, Schuit AJ, et al. (2007) Physical activity and breast cancer: A systematic review. Epidemiology 18: 137157. doi:10.1097/01.ede.0000251167.75581.98.

30. Blair SN, Kohl HW 3rd, Barlow CE, Paffenbarger RS Jr, Gibbons LW, et al (1995) Changes in physical fitness and all-cause mortality. A prospective study of healthy and unhealthy men. JAMA 273: 1093-1098.

31. van Poppel MN, Chinapaw MJ, Mokkink LB, van Mechelen W, Terwee CB (2010) Physical activity questionnaires for adults: A systematic review of measurement properties. Sports Med 40: 565-600. doi:10.2165/11531930. 